\title{
BMJ Open Evaluating associations of joint swelling, joint stiffness and joint pain with physical activity in first-degree relatives of patients with rheumatoid arthritis: Studies of the Aetiology of Rheumatoid Arthritis (SERA), a prospective cohort study
}

Jan M Hughes-Austin (D , ${ }^{1}$ Joachim H Ix, ${ }^{2}$ Samuel R Ward, ${ }^{1,3}$ Michael H Weisman, ${ }^{4}$ James R ODell, ${ }^{5}$ Ted R Mikuls, ${ }^{5}$ Jane H Buckner (1) , ${ }^{6}$ Peter K Gregersen, ${ }^{7}$ Richard M Keating, ${ }^{8} \mathrm{M}$ Kristen Demoruelle, ${ }^{9}$ Kevin D Deane, ${ }^{9} \mathrm{~V}$ Michael Holers, ${ }^{9}$ Jill M Norris ${ }^{10}$

To cite: Hughes-Austin JM, Ix JH, Ward SR, et al. Evaluating associations of joint swelling, joint stiffness and joint pain with physical activity in firstdegree relatives of patients with rheumatoid arthritis: Studies of the Aetiology of Rheumatoid Arthritis (SERA), a prospective cohort study. BMJ Open 2021;11:e050883. doi:10.1136/ bmjopen-2021-050883

- Prepublication history and additional supplemental material for this paper are available online. To view these files, please visit the journal online (http://dx.doi.org/10.1136/ bmjopen-2021-050883).

Received 23 March 2021 Accepted 09 August 2021

Check for updates

(C) Author(s) (or their employer(s)) 2021. Re-use permitted under CC BY-NC. No commercial re-use. See rights and permissions. Published by BMJ.

For numbered affiliations see end of article.

Correspondence to Dr Jan M Hughes-Austin; jhughesaustin@health.ucsd.edu

\section{ABSTRACT}

Objective Physical activity (PA) in preclinical rheumatoid arthritis (RA) is associated with lower RA risk and disease severity. As joint signs and symptoms of inflammatory arthritis serve as a barrier to PA in RA, it is important to consider whether they affect $P A$ in the time prior to $R A$. Therefore, we investigated whether joint swelling, stiffness or pain were associated with PA in first-degree relatives (FDRs) of patients with RA, a population at higher risk for future RA.

Design Prospective study design.

Setting We recruited FDRs of patients with RA from academic centres, Veterans' hospitals and rheumatology clinics or through responses to advertising from six sites across the USA.

Participants We evaluated associations of joint stiffness, joint swelling and joint pain with PA time in 268 FDRs with $\geq 2$ visits over an average 1.2 years. Clinicians confirmed joint swelling. Participants self-reported joint stiffness and/or pain.

Primary outcome measures PA during a typical 24-hour day was quantified via questionnaire, weighted to reflect metabolic expenditure, where 24 hours was the minimum PA time. Linear mixed models evaluated associations between symptoms and change in PA over time, adjusting for age, sex, race, body mass index, smoking and RArelated autoantibodies.

Results Average weighted PA time was $37 \pm 7$ hours. In the cross-sectional analysis, PA time was $1.3 \pm 0.9$ hours higher in FDRs reporting joint pain ( $p=0.15)$; and $0.8 \pm 1.6$ and $0.4 \pm 1$ hours lower in FDRs with joint swelling $(p=0.60)$ and stiffness $(p=0.69)$, respectively. Longitudinally, adjusting for baseline PA time, baseline symptoms were not significantly associated with changes in PA time. However, on average over time, joint stiffness and pain were associated with lower PA time

\section{Strengths and limitations of this study}

This study is the first to investigate whether joint swelling or related symptoms are associated with physical activity in a population of first-degree relatives (FDRs) of patients with rheumatoid arthritis (RA) who do not have RA themselves, but have higher risk of $\mathrm{RA}$.

- The physical activity questionnaire captured activity on a 'typical day' and did not fully account for fluctuations of physical activity that could have been influenced temporarily by inflammatory joint signs and symptoms, illness or other factors such as season of the year.

- Because we investigated only FDRs of patients with RA, we are not able to generalise these results to either patients living with RA, or to the general population.

$\left(p_{\text {interaction }}=0.0002, p_{\text {interaction }}=0.002\right)$, and joint swelling was associated with higher PA time $\left(\mathrm{p}_{\text {interaction }}<0.0001\right)$.

Conclusion Baseline symptoms did not predict future PA time, but on average over time, joint symptoms influenced PA time.

\section{INTRODUCTION}

Rheumatoid arthritis (RA) is a chronic systemic inflammatory disease that if untreated, leads to pain, joint destruction, disability and reduced life expectancy. Physical activity (PA) is encouraged in RA to facilitate joint mobility, physical function and mitigate cardiovascular disease ${ }^{1}$; yet it is significantly less than in individuals without 
RA, ${ }^{23}$ where muscle strength just exceeds thresholds needed to maintain activities of daily living. ${ }^{2}{ }^{3}$ Several indicators of RA disease activity, such as joint flares, ${ }^{4}$ pain level and fatigue, reduced mobility and stiffness, ${ }^{25}$ have been inversely associated and recognised as barriers to PA engagement. ${ }^{245}$

PA prior to RA has been associated with lower risk for RA, a milder disease course and less severe joint destruction. ${ }^{67}$ Thus, PA could extend the time until RA becomes clinically manifest, and presents a key opportunity to intervene in the preclinical period. Further, beyond prevention, if individuals are diagnosed with RA at a time when they have more PA, ability and strength, it may lead to them maintaining PA and independent living during the course of the disease.

As joint signs and symptoms of inflammatory arthritis serve as a barrier to PA in RA, it is important to consider whether they affect PA in the time prior to RA. Preclinical RA is characterised by the development of autoantibodies, circulating inflammatory markers, and signs and symptoms of inflammatory joint disease. ${ }^{8-10}$ Among individuals who are at risk for $\mathrm{RA}$, such as first-degree relatives (FDRs) of patients with RA, and those who may exhibit preclinical RA features, it is not known whether inflammatory joint signs and symptoms are associated with more or less PA time. Therefore, we hypothesised a priori that inflammatory joint symptoms such as joint swelling, joint stiffness and joint pain would be associated with less PA time. Using FDRs of patients with RA from the Studies of the Aetiology of Rheumatoid Arthritis (SERA), a population without RA but at increased risk for RA, we sought to determine whether joint swelling, joint stiffness and joint pain are associated with PA time at baseline and over time.

\section{METHODS}

\section{Studies of the Aetiology of Rheumatoid Arthritis}

Founded in 2003, SERA is a multicentre study designed to examine the role of environmental and genetic factors in the development and progression of RA-related autoimmunity, and to explore preclinical immunological changes and pathophysiological processes in the absence of treatments or secondary complications of active RA disease. ${ }^{9}$ The SERA cohort consists of FDRs (parent, sibling or offspring) of probands with RA, who are selected for this prospective study because of higher RA risk. FDRs were recruited through probands (identified from academic centres, Veterans' hospitals and rheumatology clinics or through responses to advertising) from six sites across the USA (Denver, Colorado, Los Angeles, California, Seattle, Washington, Manhasset, New York, Omaha, Nebraska and Chicago, Illinois). FDRs were eligible to participate if they did not meet the 1987 American College of Rheumatology (ACR) Classification Criteria for RA, ${ }^{11}$ and were $\geq 18$ years old. At research visits, FDRs completed disease and exposure assessment questionnaires, underwent a standardised interview and 68-count joint examination by a trained study clinician, and had blood drawn.
FDRs positive for any RA-related autoantibody at visits were seen annually while autoantibody negative FDRs were seen every 2 years. Institutional Review Boards at each of the SERA sites approved this study, and all participants provided written informed consent.

\section{Patient and public involvement}

While no patients or members of the public were involved in the planning of the study, participants in this study were provided the results of their autoantibody testing as well as study updates via a regular newsletter.

\section{Study population}

In SERA, PA questions were not routinely asked until 2010, so we used the first time an FDR answered this question, between 2010 and 2014, as their 'baseline' visit. There were 878 FDRs seen in the research clinic within this 4-year period who had complete information regarding PA and the presence of joint swelling, joint stiffness or joint pain. Of these 878 FDRs, 268 FDRs had $\geq 2$ research clinic visits, which allowed us to also investigate changes in PA over time. There were no significant differences between this subset of 268 FDRs and the larger cohort of 878 FDRs with regard to demographics and clinical characteristics. These 268 FDRs with multiple research clinic visits comprised our analysis dataset.

\section{RA-related inflammatory joint swelling or symptoms}

Trained clinicians, blinded to autoantibody status, confirmed the presence of joint swelling using a 68-count joint examination during research clinic visits. Additional inflammatory joint symptoms involving the same joints were indicated by the presence of self-reported joint stiffness and pain during the same research clinic visit. Consistent with Sparks et al, we included joints at RA-specific sites according to the ACR/European League Against Rheumatism (EULAR) 2010 classification for RA, that is, any of the metacarpophalangeal, proximal interphalangeal or metatarsophalangeal joints, wrist, as well as the elbow due to its relative specificity for RA. ${ }^{12} 13$

\section{Physical Activity (PA) time}

Hours sleeping, sitting and performing slight (eg, standing, walking), moderate (eg, household work) or heavy (eg, heavy labour, intense exercise) activity during a typical 24-hour period were obtained through questionnaire administered during research clinic visits, and were measured as a continuous score (online supplemental table 1). PA hours were weighted to reflect metabolic expenditure, calculated as: PA Score $=1.0 * \mathrm{~h}_{\text {sleep }}+1.1 * \mathrm{~h}_{\text {sed- }}$ entary $+1.5 * \mathrm{~h}_{\text {slight }}+2.4 * \mathrm{~h}_{\text {moderate }}+5.0 * \mathrm{~h}_{\text {heavy }}$, with 24 hours representing the minimum possible score. For example, an individual who slept 8 hours, was sedentary for 9 hours, performed slight activity for 4 hours, moderate activity for 2 hours and heavy activity for 1 hour had a weighted PA score of 33.7 hours for that day. This PA score has been validated and used previously. ${ }^{14}$ 


\section{Covariates}

Participants also underwent a complete examination and completed additional questionnaires to obtain age, race/ethnicity, smoking history (current vs not current and pack-years), weight (kilograms) and height (metres), which allowed us to calculate body mass index (BMI, $\left.\mathrm{kg} / \mathrm{m}^{2}\right)$. Serum was also collected and used to measure RA-related autoantibodies at the time of the visit, specifically rheumatoid factor (RF) measured by nephelometry, RF isotypes, IgM, IgG and IgA, as well as anticyclic citrullinated peptide antibodies (anti-CCP2 and anti-CCP3.1) as described previously. ${ }^{9}$

\section{Statistical analysis}

We evaluated differences in demographics, autoantibody status, inflammatory markers and PA time in FDRs with and without joint swelling or inflammatory joint symptoms using Student's t-test or Wilcoxon rank-sum test for continuous variables and $\chi^{2}$ test or Fisher's exact test for categorical variables. We used a cross-sectional study design with analysis of covariance among 268 FDRs to examine whether baseline examination inflammatory joint symptoms were associated with baseline PA time, adjusting for age, sex, race, BMI, smoking and positivity for RA-related autoantibodies. We then used a prospective study design to evaluate whether baseline joint swelling or symptoms were associated with PA over time, and whether changes in joint swelling or symptoms were associated with changes in PA over time. For the longitudinal analyses, we used linear mixed models, and adjusted for age, sex, race, BMI, smoking and positivity for RA-related autoantibodies. To determine whether changes in joint symptoms were associated with changes in PA over time, we adjusted for baseline PA and incorporated an interaction term with time into the model, as it indicated whether there was an effect of time on the association between joint symptoms and PA hours, accounting for multiple visits per FDR. We also performed a sensitivity analysis for upper extremity joint swelling, stiffness and pain, as well as for lower extremity joint swelling, stiffness and pain following the above methods. All analyses were conducted in SAS V.9.4 (SAS Institute) and values of $\mathrm{p}<0.05$ were considered statistically significant.

\section{RESULTS}

The 268 FDRs in this study had mean age of $50 \pm 15$ years at baseline, BMI of $28 \pm 10 \mathrm{~kg} / \mathrm{m}^{2}$ and a mean $37 \pm 7$ weighted PA hours per day. Table 1 presents participant characteristics, stratified by present joint swelling. At baseline, 20 (7\%) FDRs had joint swelling, 50 (19\%) reported joint stiffness and $84(31 \%)$ reported joint pain. These groups were not mutually exclusive with $11 \%$ reporting joint stiffness and pain, 3\% with joint swelling and reporting pain, $0 \%$ with joint swelling and reporting stiffness, and $2 \%$ reporting all three-joint swelling, stiffness and pain. FDRs with joint swelling, compared with FDRs without joint swelling, were significantly older and were more
Table 1 Descriptive statistics by joint swelling at the baseline examination in 268 FDRs of patients with RA

\begin{tabular}{|c|c|c|c|}
\hline & $\begin{array}{l}\text { Joint } \\
\text { swelling } \\
(\mathrm{n}=20)\end{array}$ & $\begin{array}{l}\text { No joint } \\
\text { swelling } \\
(n=248)\end{array}$ & $P$ value \\
\hline Age, years (SD) & $58(17)$ & $49(15)$ & 0.01 \\
\hline Female, \% & 60 & 71 & 0.28 \\
\hline Race, \% non-Hispanic white & 90 & 81 & $0.54^{*}$ \\
\hline $\mathrm{BMI}, \mathrm{kg} / \mathrm{m}^{2}$, mean (SD) & $27(6)$ & $28(10)$ & 0.53 \\
\hline RF, \% positive & 10 & 14 & $1.00^{*}$ \\
\hline CCP2, \% positive & 5 & 3 & $0.47^{*}$ \\
\hline CCP3.1, \% positive & 17 & 9 & $0.33^{*}$ \\
\hline RF IgM, \% positive & 0 & 13 & $0.08^{\star}$ \\
\hline RF IgG, \% positive & 10 & 28 & $0.11^{*}$ \\
\hline RF IgA, \% positive & 10 & 3 & $0.17^{*}$ \\
\hline \multicolumn{4}{|c|}{ No of autoantibodies, median (25th, 75 th percentile) } \\
\hline $\begin{array}{l}\text { CRP, median (25th, 75th } \\
\text { percentile) }\end{array}$ & $2(1,3)$ & $1(1,4)$ & $0.91 \dagger$ \\
\hline Ever smoke, \% & 25 & 15 & $0.34^{*}$ \\
\hline $\begin{array}{l}\text { Pack-years smoking, median } \\
\text { (25th, 75th percentile) }\end{array}$ & $0(0,12)$ & $0(0,4)$ & $0.26 \dagger$ \\
\hline $\begin{array}{l}\text { Education greater than high } \\
\text { school, \% }\end{array}$ & 100 & 97 & $1.00^{*}$ \\
\hline \multicolumn{4}{|c|}{ Physical activity, hours, mean (SD) } \\
\hline Sleep & $7(1)$ & $7(1)$ & 0.21 \\
\hline Sedentary & $7(3)$ & $7(3)$ & 0.45 \\
\hline Slight & $5(2)$ & $5(3)$ & 0.25 \\
\hline Moderate & $4(3)$ & $3(2)$ & 0.17 \\
\hline Heavy & $1(1)$ & $1(2)$ & 0.17 \\
\hline $\begin{array}{l}\text { Weighted physical activity } \\
\text { hours, mean (SD) }\end{array}$ & $36(5)$ & $37(7)$ & 0.71 \\
\hline
\end{tabular}

Values in this table are presented as means (and SD) or as medians (and 25th and 75th percentile); and as proportions of FDRs with the specified characteristic.

*Indicates use of Fisher's exact test for $p$ values.

†Indicates use of Wilcoxon rank-sum test for $p$ values.

BMI, body mass index; CCP, cyclic citrullinated peptide; CRP, C-

Reactive Protein; FDRs, first-degree relatives; RA, rheumatoid arthritis; $\mathrm{RF}$, rheumatoid factor.

likely to be anti-CCP2 and anti-CCP3.1 positive, and had slightly less PA time, a difference that did not achieve statistical significance. FDRs with joint stiffness and pain, however, had similar age, had similar autoantibody profiles and similar PA time compared with FDRs without joint stiffness and pain (online supplemental tables 2 and 3).

Cross-sectional analysis of inflammatory joint symptoms at baseline showed that after adjusting for age, sex, race, BMI, pack-years smoking and positivity for RA-related autoantibodies, FDRs reporting joint pain had 1.3 more PA hours than FDRs without joint pain (38.1 \pm 0.9 vs $36.8 \pm 0.6$ PA hours, respectively; $95 \%$ CI: -0.46 to 3.06, $\mathrm{p}=0.15$ ), although this association was not statistically significant. In contrast, FDRs self-reporting joint stiffness had 0.4 fewer PA hours than FDRs without joint stiffness 


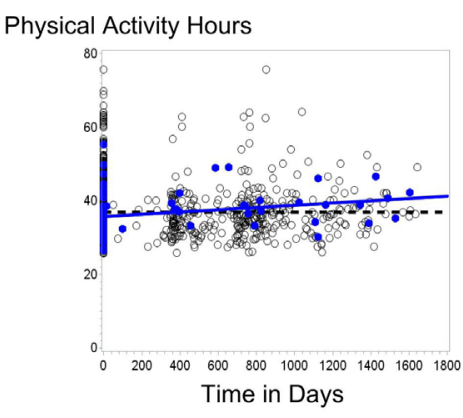

A Joint swelling at baseline pinteraction $=0.33$

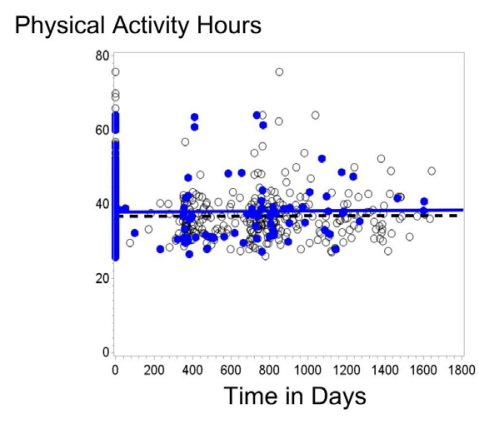

B Joint stiffness at baseline pinteraction $=0.33$

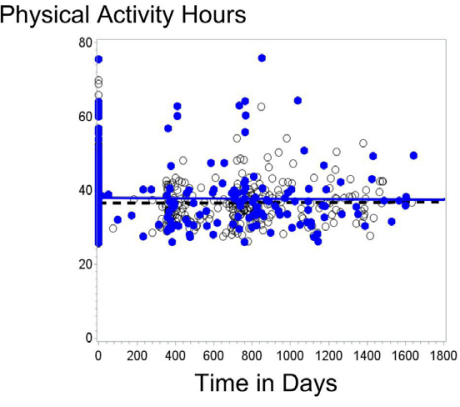

C Joint pain at baseline Pinteraction $=0.99$

\section{Symptoms Present}

Symptoms Absent

Figure 1 Associations between baseline joint swelling, stiffness and pain, and physical activity over time in 268 FDRs of patients with RA. Figure 1 shows weighted physical activity hours over time in days for FDRs with joint swelling (A), joint stiffness (B) and joint pain (C) at baseline, indicated by blue dots and blue solid lines, compared with FDRs without joint symptoms at baseline, indicated by black dots and black dashed lines. Analyses were fully adjusted for baseline physical activity hours, age, sex, race, pack-years of smoking, body mass index and being positive for RA-related autoantibodies. FDRs, first-degree relatives; RA, rheumatoid arthritis.

(36.8 \pm 1.1 vs $37.3 \pm 0.6$ PA hours, respectively; $95 \% \mathrm{CI}$ : -2.51 to 1.66, $\mathrm{p}=0.69$ ); and FDRs with joint swelling documented by examination had 0.8 fewer PA hours than FDRs without joint swelling (36.4 \pm 1.6 vs $37.3 \pm 0.6 \mathrm{PA}$ hours, respectively; $95 \%$ CI: -3.96 to 2.29 , $\mathrm{p}=0.60$ ) in fully adjusted analysis.

The average length of follow-up was 1.2 years (range 14 days to 4.5 years). In this longitudinal analysis, adjusting for baseline PA time, age, sex, race, pack-years smoking, autoantibody status and BMI, baseline joint symptoms were not significantly associated with a change in PA hours over time (figure 1).

Longitudinal analysis evaluating the interaction between joint signs and symptoms and PA over time showed in fully adjusted models that FDRs with subsequent occurrences of joint swelling had more PA hours over time than FDRs without joint swelling ( $p_{\text {interaction }}<0.0001 ; \beta=0.004 \pm 0.001$, $\mathrm{p}<0.0001)$. Conversely, FDRs reporting joint stiffness in subsequent research clinic visits had fewer PA hours over time compared with FDRs who did not report joint stiffness $\left(\mathrm{p}_{\text {interaction }}=0.0002 ; \beta=-0.001 \pm 0.001, \mathrm{p}=0.11\right)$. Similarly, FDRs reporting joint pain in subsequent research clinic visits had fewer PA hours over time compared with FDRs not reporting joint pain $\left(\mathrm{p}_{\text {interaction }}=0.002 ; \beta=-0.001 \pm 0.001\right.$, $\mathrm{p}=0.32$ ) (figure 2).

Findings from the sensitivity analysis for upper extremity joint swelling resembled the results from joint swelling, where in adjusted analysis, FDRs with upper

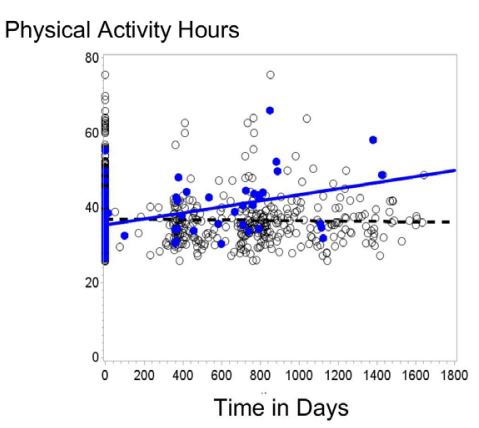

A Joint swelling occurrences over time $p_{\text {interaction }}<0.0001$

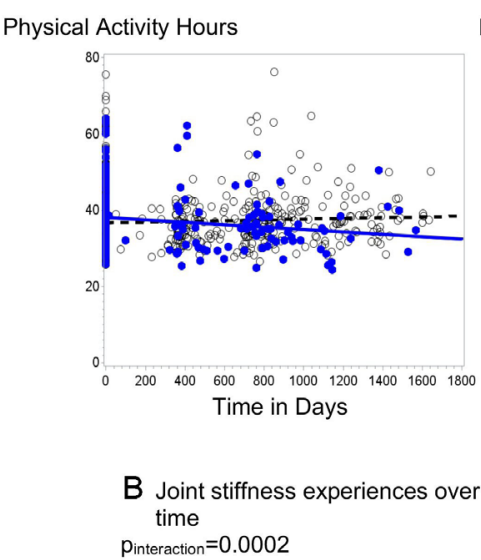

Pinteraction $=0.0002$

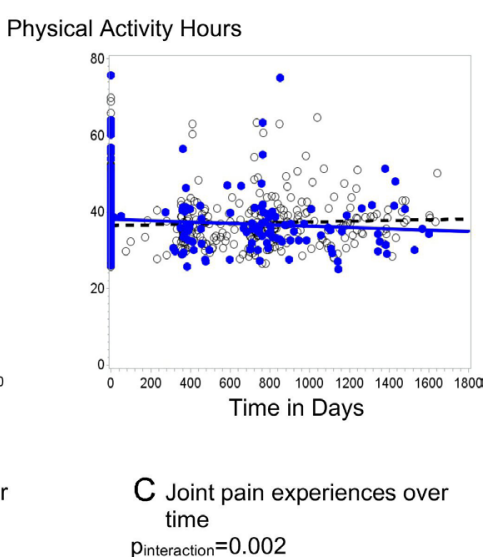

Pinteraction $=0.002$

Symptoms Present

Symptoms Absent $\bigcirc$

Figure 2 Associations between changes in joint swelling, stiffness, and pain, and physical activity over time in 268 FDRs of patients with RA. Figure 2 shows weighted physical activity hours over time in days for FDRs with joint swelling (A), joint stiffness (B) and joint pain (C) during subsequent research clinic visits, indicated by blue dots and blue solid lines, compared with FDRs without joint symptoms at subsequent research clinic visits, indicated by black dots and black dashed lines. Analyses were fully adjusted for baseline physical activity hours, age, sex, race, pack-years of smoking, body mass index and being positive for RA-related autoantibodies. FDRs, first-degree relatives; RA, rheumatoid arthritis. 
extremity joint swelling had more PA hours over time than FDRs without upper extremity joint swelling $\left(\mathrm{p}_{\text {inter- }}\right.$ action $<0.0001 ; \beta=0.003 \pm 0.001, p=0.003$ ). Neither upper or lower extremity stiffness nor pain was associated with PA time in any of the sensitivity analyses.

\section{DISCUSSION}

In FDRs of patients with RA who were free of RA, we observed associations between joint swelling, joint stiffness and joint pain and PA over time. Taking into account baseline PA hours, joint symptoms at baseline were not associated with PA hours over time. As FDRs developed joint symptoms over time, however, PA hours changed. On average, FDRs reporting joint stiffness and pain had less PA, and FDRs experiencing joint swelling had more PA, independent of age, sex, race, pack-years of smoking, BMI and being positive for RA-related autoantibodies. These findings suggest current joint symptoms do not predict future PA, but that over time, average PA is influenced by joint symptoms. The inverse associations we found between joint stiffness and joint pain with PA are consistent with previous findings that RA flares and activity limitation are inversely associated with PA in patients with RA; $;^{25}$ and indicate that similar joint involvement in a population without RA, but at higher risk for RA, may experience similar disease-specific barriers to PA as patients with active RA.

PA is a robust predictor of multiple health outcomes such as diabetes, hypertension and cardiovascular disease. Recent exciting and important findings suggest that PA prior to RA development has been associated with a lower risk of RA, a milder disease course and less joint destruction. ${ }^{67}$ In particular, increased time in selfreported PA 5 years before RA diagnosis was associated with decreased disease activity, joint pain and inflammatory marker levels, as well as better self-reported health following disease onset. ${ }^{7}$ Despite these benefits, patients with RA have lower PA levels than their counterparts without RA. ${ }^{2}$ It has been shown that patients with RA average 1000 fewer steps per day during a flare period compared with a non-flare period. ${ }^{4}$ Yet among individuals who had established health-enhancing PA, their PA levels remained consistent even during periods of high RA disease activity. ${ }^{15}$ Given the modest results from our study, future directions for research in this population of FDRs include addressing joint stiffness and pain during this preclinical period. By addressing these symptoms, clinicians could potentially provide individuals at risk for RA the tools to establish and sustain regular PA prior to and potentially during the onset of RA, which could lead to the ability to maintain physical function and independent living during the course of the disease.

This study has important limitations. We were limited in our estimation of PA as particular tasks, for example, walking versus rock climbing were not collected in this questionnaire. RA usually begins in the small joints of the hands and feet, and spreads later to the larger joints, as the articular cartilage and bone erode. Therefore, we chose to focus on the small joints of the hands and feet, as well as the elbow, in order to identify joint swelling and/or symptoms most indicative of earlier processes. In sensitivity analysis, however, we investigated whether upper extremity joint symptoms vs lower extremity joint symptoms were associated with PA hours. These findings were consistent with our primary findings, and suggest that PA hours did not differ based on whether joint symptoms were predominantly in the upper or lower extremities. The PA questionnaire captured activity on a 'typical day' and did not fully account for fluctuations of PA that could have been influenced temporarily by inflammatory joint signs and symptoms, illness or other factors such as season of the year. Thus, our findings captured an overall picture of PA that likely underestimated associations between joint symptoms and PA time. Finally, because we investigated only FDRs of patients with RA, we are not able to generalise these results to either patients living with RA, or to the general population.

This study is the first to our knowledge to investigate whether joint swelling or related symptoms are associated with PA in a population of FDRs of patients with RA who do not have RA themselves, but have higher risk of RA. The associations between joint stiffness and pain and PA were consistent with our hypothesis and prior literature, whereas the association between joint swelling and PA was in a direction opposite to what we hypothesised. Future studies are needed to determine if symptoms of inflammatory arthritis are primary barriers to PA and whether intervening on the symptoms will allow continued pursuit of PA during this preclinical RA period.

In conclusion, among FDRs of patients with RA, joint swelling, joint stiffness and joint pain at baseline were not associated with future PA time. As joint swelling or symptoms changed over time, however, so did time in PA. Given these results, implications include adapting exercise and daily PA for individuals with joint pain or stiffness in order to intervene before the clinical manifestation of RA and potentially extend the period where they are RA-free.

\section{Author affiliations}

${ }^{1}$ Department of Orthopaedic Surgery, University of California, San Diego, La Jolla, California, USA

${ }^{2}$ Department of Medicine, Division of Nephrology-Hypertension, University of California, San Diego, La Jolla, California, USA

${ }^{3}$ Department of Radiology, University of California, San Diego, La Jolla, California, USA

${ }^{4}$ School of Medicine, Division of Rheumatology, Cedars-Sinai Medical Center, Los Angeles, California, USA

${ }^{5}$ College of Medicine, Division of Rheumatology, University of Nebraska Medical Center, Omaha, Nebraska, USA

${ }^{6}$ Translational Research Institute, Benaroya Research Institute at Virginia Mason, Seattle, Washington, USA

${ }^{7}$ Roberts S Boas Center for Genomics and Human Genetics, Feinstein Institute for Medical Research, Manhasset, New York, USA

${ }^{8}$ Division of Rheumatology, Scripps Green Hospital, La Jolla, California, USA

${ }^{9}$ School of Medicine, Department of Rheumatology, University of Colorado -

Anschutz Medical Campus, Aurora, Colorado, USA 
${ }^{10}$ Department of Epidemiology, Colorado School of Public Health, University of Colorado - Anschutz Medical Campus, Aurora, Colorado, USA

Acknowledgements The authors thank the SERA participants, investigators and staff for their valuable contributions to this research.

Contributors JMH-A contributed to the conceptualisation, methodology, data curation, formal analysis, investigation, writing original draft, writing-review and editing, funding acquisition. JHI contributed to the investigation, writing original draft, writing-review and editing. SRW contributed to the conceptualisation, investigation, writing-review and editing, funding acquisition. MMW, JRO, TRM, JHB, PKG, RMK, MKD and VMH contributed to the methodology, data curation, investigation, writing-review and editing. KDD contributed to the conceptualisation, methodology, data curation, investigation, writing-review and editing. JMN contributed to the methodology, formal analysis, investigation, writing original draft, writing-review and editing, funding acquisition.

Funding This work was supported by the Foundation for Physical Therapy (Miami-Marquette Challenge Research Grant), the National Institute of Arthritis and Musculoskeletal and Skin Diseases (R01 AR051394-05), National Heart, Lung and Blood Institute (K01HL122394) and National Institute for Diabetes and Digestive and Kidney Diseases (K24DK110427) at the National Institutes of Health, the ACR Rheumatology Research Foundation (Within Our Reach: Finding a Cure for Rheumatoid Arthritis campaign, and the Career Development Bridge Funding Award: K Bridge), and the Autoimmunity Prevention Center (U19 Al050864-10) at the National Institutes of Health.

Competing interests None declared.

Patient consent for publication Not required.

Ethics approval This project is a secondary analysis of existing data in SERA. JMH-A obtained IRB approval from University of California, San Diego to analyze the data. (UCSD HRPP 150004). Each SERA site listed here obtained approval from their respective review boards: Colorado Multiple Institutional Review Board, Cedar Sinai Institutional Review Board, Benaroya Research Institute Research Protections Department, Feinstein Institutes for Medical Research Human Research Protection Programme, University of Nebraska Medical Center Institutional Review Board, The Biological Sciences Division/University of Chicago Medical Center Institutional Review Board.

Provenance and peer review Not commissioned; externally peer reviewed.

Data availability statement Data are available upon reasonable request. Data are currently not in a repository, but are available upon request from the Studies of the Etiology of Rheumatoid Arthritis (SERA). Please contact the corresponding author, JMH-A, at jhughesaustin@health.ucsd.edu to relay your request to SERA.

Supplemental material This content has been supplied by the author(s). It has not been vetted by BMJ Publishing Group Limited (BMJ) and may not have been peer-reviewed. Any opinions or recommendations discussed are solely those of the author(s) and are not endorsed by BMJ. BMJ disclaims all liability and responsibility arising from any reliance placed on the content. Where the content includes any translated material, BMJ does not warrant the accuracy and reliability of the translations (including but not limited to local regulations, clinical guidelines, terminology, drug names and drug dosages), and is not responsible for any error and/or omissions arising from translation and adaptation or otherwise.

Open access This is an open access article distributed in accordance with the Creative Commons Attribution Non Commercial (CC BY-NC 4.0) license, which permits others to distribute, remix, adapt, build upon this work non-commercially, and license their derivative works on different terms, provided the original work is properly cited, appropriate credit is given, any changes made indicated, and the use is non-commercial. See: http://creativecommons.org/licenses/by-nc/4.0/.

ORCID iDs

Jan M Hughes-Austin http://orcid.org/0000-0002-7255-274X

Jane H Buckner http://orcid.org/0000-0002-9005-1885

\section{REFERENCES}

1 Metsios GS, Stavropoulos-Kalinoglou A, Kitas GD. The role of exercise in the management of rheumatoid arthritis. Expert Rev Clin Immunol 2015;11:1121-30.

2 Sharif K, Watad A, Bragazzi NL, et al. Physical activity and autoimmune diseases: get moving and manage the disease. Autoimmun Rev 2018;17:53-72.

3 Sokka T, Häkkinen A, Kautiainen $\mathrm{H}$, et al. Physical inactivity in patients with rheumatoid arthritis: data from twenty-one countries in a cross-sectional, International study. Arthritis Rheum 2008:59:42-50

4 Jacquemin C, Molto A, Servy H, et al. Flares assessed weekly in patients with rheumatoid arthritis or axial spondyloarthritis and relationship with physical activity measured using a connected activity tracker: a 3-month study. RMD Open 2017;3:e000434.

5 Veldhuijzen van Zanten JJCS, Rouse PC, Hale ED, et al. Perceived barriers, facilitators and benefits for regular physical activity and exercise in patients with rheumatoid arthritis: a review of the literature. Sports Med 2015;45:1401-12.

6 Di Giuseppe D, Bottai M, Askling J, et al. Physical activity and risk of rheumatoid arthritis in women: a population-based prospective study. Arthritis Res Ther 2015;17:40.

7 Sandberg MEC, Wedrén S, Klareskog L, et al. Patients with regular physical activity before onset of rheumatoid arthritis present with milder disease. Ann Rheum Dis 2014;73:1541-4.

8 Hughes-Austin JM, Deane KD, Derber LA, et al. Multiple cytokines and chemokines are associated with rheumatoid arthritis-related autoimmunity in first-degree relatives without rheumatoid arthritis: studies of the aetiology of rheumatoid arthritis (SERA). Ann Rheum Dis 2013;72:901-7.

9 Kolfenbach JR, Deane KD, Derber LA, et al. A prospective approach to investigating the natural history of preclinical rheumatoid arthritis (RA) using first-degree relatives of probands with RA. Arthritis Rheum 2009;61:1735-42.

10 Young KA, Deane KD, Derber LA, et al. Relatives without rheumatoid arthritis show reactivity to anti-citrullinated protein/peptide antibodies that are associated with arthritis-related traits: studies of the etiology of rheumatoid arthritis. Arthritis Rheum 2013;65:1995-2004.

11 Arnett FC, Edworthy SM, Bloch DA, et al. The American rheumatism association 1987 revised criteria for the classification of rheumatoid arthritis. Arthritis Rheum 1988;31:315-24.

12 Sparks JA, Chang S-C, Deane KD, et al. Associations of smoking and age with inflammatory joint signs among unaffected firstdegree relatives of rheumatoid arthritis patients: results from studies of the etiology of rheumatoid arthritis. Arthritis Rheumatol 2016;68:1828-38.

13 Aletaha D, Neogi T, Silman AJ, et al. 2010 rheumatoid arthritis classification criteria: an American College of Rheumatology/ European League against rheumatism collaborative initiative. Arthritis Rheum 2010;62:2569-81.

14 Shortreed SM, Peeters A, Forbes AB. Estimating the effect of long-term physical activity on cardiovascular disease and mortality: evidence from the Framingham heart study. Heart 2013;99:649-54.

15 Demmelmaier I, Dufour AB, Nordgren B, et al. Trajectories of physical activity over two years in persons with rheumatoid arthritis. Arthritis Care Res 2016;68:1069-77. 